\title{
Successful management of fetal atrial flutter at term pregnancy with postnatal electrocardioversion.
}

\author{
Nnadozie Igbokwe ${ }^{1}$, Aisha Firzana Ibrahim ${ }^{1}$, Samy Mutalab $^{1}$, and Oonagh Cleland ${ }^{1}$ \\ ${ }^{1}$ Royal Jubilee Maternity Service
}

May 11, 2021

\begin{abstract}
Fetal Atrial flutter (AF) is an uncommon condition accounting for about $30 \%$ of all fetal tachyarrhythmias. It is associated with structural heart anomalies and hydrops, with $10 \%$ fetal mortality rate. This case demonstrates a successfully managed atrial flutter at term with postnatal electrocardioversion using multidisciplinary team approach.
\end{abstract}

\section{Title}

Successful management of fetal atrial flutter at term pregnancy with postnatal electrocardioversion.

Authors

Nnadozie Igbokwe (MBBS, MMedSci, MRCPI, MRCOG) corresponding author

Education Fellow for medical students, Queens University Belfast, UK

ST5 Trainee Registrar in Obstetrics and Gynaecology, Royal Jubilee Maternity Hospital, UK

Email: dozzybarry4@gmail.com

Aisha F Ibrahim (MRes, MB BCh BAO)

ST1 Trainee doctor, Obstetrics and Gynaecology

Royal Jubilee Maternity Hospital

Email: AishaBinti.ibrahim@belfasttrust.hscni.net

Samy Mutalab (MD)

Locum Consultant Obstetrician and Gynaecologist

Royal Jubilee Maternity Hospital

Email: samy.mutalab@belfasttrust.hscni.net

Oonagh Cleland (MRCOG)

Consultant Obstetrician and Gynaecologist

Royal Jubilee Maternity Hospital

Email: Oonagh.cleland@belfasttrust.hscni.net

\section{Abstract}


Fetal Atrial flutter (AF) is an uncommon condition accounting for about $30 \%$ of all fetal tachyarrhythmias. It is associated with structural heart anomalies and hydrops, with $10 \%$ fetal mortality rate.

This case demonstrates a successfully managed atrial flutter at term with postnatal electrocardioversion using multidisciplinary team approach.

\section{Key Message}

At term pregnancy, emergency delivery and postnanal electrocardioversion with prophylactic digitalisation is a successful treatment option for fetal AF. Clinicians should be aware of AF and its diagnosis, and not just manage it as fetal distress.

\section{Introduction}

Fetal AF is a serious and threatening form of tachyarrhythmia, as it may cause fetal hydrops and associated with fetal mortality and neurological complications. The incidence of fetal tachyarrhythmia in less than $1 \%$ of pregnancies, and the two commonest forms are AF (10-30\%) and supraventricular tachycardia (SVT) $(66-90 \%){ }^{1}$

Fetal AF is characterised by a rapid regular atrial contraction (300-600 beats/minute). ${ }^{2}$ This makes the ventricles unable to respond to this rapid speed in a 1:1 fashion leading to a $2: 1$ or variable atrioventricular (AV) block or 2:1 conduction. It commonly develops in the third trimester and should not be confused with fetal tachycardia or distress. The proposed underlying mechanism causing fetal AF is the reentrant circuit causing premature atrial impulses. ${ }^{3}$ The use of M-mode ultrasound establishes the diagnosis followed by fetal echocardiogram to rule out structural heart anomalies. The outcome of fetal tachyarrhythmia is dependent on the presence of hydrops and cardiac disease, and not the type of tachyarrhythmia. ${ }^{4}$ Treatment is individualised with factors like gestational age, structural heart problems and hydrops taken into significant consideration. Early detection and treatment improved clinical outcome significantly. ${ }^{5}$

We present a case of fetal atrial flutter in a 24 -year-old low risk pregnancy at 37 weeks gestation. There was no fetal history of hydrops or cardiac disease and she had emergency caesarean section. Neonatal sinus rhythm was restored with single electrocardioversion and some course of digitalisation.

\section{Case Report}

A 24-year-old primigravida, booked low risk in a different hospital. Her booking blood results ultrasound scan were normal with Haemoglobin level of $144 \mathrm{~g} / \mathrm{L}$, and she had a normal anomaly scan at 20 weeks gestation. She had regular uneventful antenatal midwife-led care and had no significant past medical history of note. At each clinic visit, the fetal heart rate (FHR) was normal in keeping with gestational age.

At 37 weeks gestation she attended her routine antenatal care, also with first episode of reduced fetal movement. There was difficulty in detecting the FHR by both the hand-held fetal Doppler and cardiotocographic (CTG) machine as the heartbeat was too fast. A quick bedside ultrasound scan showed FHR of 220 beats per minute (bpm). The maternal pulse was $100 \mathrm{bpm}$ with blood pressure of $128 / 76 \mathrm{mmHg}$. She was quickly transferred by ambulance to the Obstetric Unit of the nearest general hospital. A quick transabdominal ultrasound scan done by the Obstetric team showed live active singleton gestation with ventricular FHR of $260 \mathrm{bpm}$ with an impression of fetal SVT made. There was a discussion to go for emergency caesarean section however this was forestalled by specialist advice from the paediatric cardiologist in a tertiary hospital who advised for the patient to be transferred by ambulance to the University teaching hospital to provide adequate neonatal care after delivery.

On arrival at the tertiary hospital 2 hours later, obstetric ultrasonography showed estimated fetal weight compatible with her gestation, with normal liquor volume and normal anterior placentation. Fetal cardiac ultrasound done by the paediatric cardiologist revealed an atrial heart rate of $480 \mathrm{bpm}$, and ventricular rate of $240 \mathrm{bpm}$ using the M-mode function. There was a mild tricuspid valve regurgitation, but no obvious cardiac structural anomaly or hydrops noted. A clinical impression of fetal AF at 480bpm with 2:1 nodal AV block was made. Options of management were discussed with the patient including medical antenatal treatment or 
abdominal delivery with neonatal management. Since the pregnancy was term, a joint agreement with the obstetric team was for urgent abdominal delivery and postnatal treatment to which the patient consented. She had an emergency caesarean section under regional block. The Outcome was a live female neonate who weighed 3530 grams at birth with favourable Apgar score although with poor colour.

The baby required some continuous positive airway pressure (CPAP) for support for a few minutes as the oxygen saturation was at $70 \%$ and was transferred immediately to the neonatal intensive care unit (NICU). ECG done showed ventricular hear beat of 235bpm (Figure 1), with persistent AF. A single synchronised direct-current cardioversion of 4 joules following ketamine sedation reverted the arrhythmia to sinus rhythm at $175 \mathrm{bpm}$. Loading dose of digoxin was started $(15 \mathrm{mcg} / \mathrm{kg})$ followed later by maintenance dose of $10 \mathrm{mcg}$ twice daily with serum digoxin level monitored. A neonatal echocardiography done on the day of delivery showed a patent foramen ovale (PFO), and mild tricuspid regurgitation with otherwise normal ventricular function.

The baby's mother had a good post-operative recovery and was well debriefed with follow up plan made. There was no neonatal recurrence of AF while on admission, and on day 4 neonatal life, the baby was discharged back to the NICU of the general hospital where she was monitored for 3 days. She was then discharged home on same dose of digoxin for prophylaxis and the last serum digoxin level before discharge was normal at $1.5 \mathrm{ug} / \mathrm{L}$. Follow up echocardiogram done 8 weeks after discharged showed similar findings as before with normal ventricular function. The baby is to be followed up every few months with an echocardiogram and review, and there has been no concern so far. Her development has been normal up to the point of writing this article at the age of 4 months.

\section{Discussion}

Fetal heart rate monitoring remains an important part of antenatal care, and as seen in our case, the tachycardia was first detected by routine fetal heart rate check. ${ }^{5}$ Fetal AF accounts for roughly 25-30 of all fetal tachyarrhythmias and is associated with variable AV conduction. There are congenital structural anomalies that may occur with AF including hypoplastic left heart syndrome, atrioventricular septal defect, pulmonary atresia and Ebstein's anomaly. Both SVT and fetal AF have similar incidence of hydrops fetalis averaging $40 \%$ and similar mortality rate of $10 \%$. Studies have shown that hydropic foetuses with fetal AF have higher ventricular rates than the non-hydropic ones, but no difference in the atrial rates. ${ }^{6}$

Most cases of fetal AF occur in the third trimester as seen in our case, with the median age presentation of 32 weeks. The ventricles are protected during fetal AF by the AV node which is not part of the intra-atrial re-entrant circuit. This is achieved by variably blocking the AV conduction, with 2:1 AV block present in over $80 \%$ of patients with fetal AF. ${ }^{4,7}$ This finding is in keeping with our patient who had 2:1 block. Fetal AF could easily be diagnosed by clinicians using the M-mode ultrasound with the apical four-chamber heart view. Both the atria and ventricles should be clearly identified in relation to the spine and descending aorta as landmarks. The focus should not be to get the ventricular rate only in fetal tachyarrhythmia using the power Doppler button. With the M-mode across both either atrium or ventricle, their respective rates could be measured and the variable conduction pattern also. It is very important to make the right diagnosis before proceeding to treatment especially in low resource setting or general hospitals without paediatric cardiologist services. In our case, the initial diagnosis was thought to be fetal SVT before the patient was referred. This is because no simultaneous atrial reading was taken. This is one of the major reasons of writing this report for educational purposes. Secondly, it is known that up to $20 \%$ of fetal tachyarrhythmia is associated with cardiac anomaly, hence the need to perform fetal echocardiography before deciding on management option..$^{3,8}$ This informs the need of multidisciplinary team management with neonatologists, paediatric cardiologist, and obstetricians. At term pregnancies, the babies should not just be delivered based on fetal distress without these considerations as the outcomes may be untoward. This is well exemplified in this case.

Based on the points highlighted above, the antenatal management of fetal AF depends on several factors including fetal gestational age, presence of fetal hydrops or features of heart failure, and associated structural 
heart disease. The risk of hydrops in fetal AF is said to be more with a ventricular rate of over $210 \mathrm{bpm}$. There was no hydrops in our case even though the ventricular heart rate was $260 \mathrm{bpm} .{ }^{6}$ The development of associated cardiac anomaly is associated with poorer neonatal outcome, hence the advantage of early detection and treatment. Although prenatal treatment of fetal AF with transplacental antiarrhythmic medications is the most common documented method of treatment, this is not always the case. ${ }^{9}$ At term or late preterm gestation, delivery of the foetus with postnatal treatment is often considered as a better choice. ${ }^{3,10}$ This obviates the adverse effects of the medication on the mother and the risk of transplacental treatment on the foetus. This explains the basis of our postnatal treatment modality.

The commonly used antiarrhythmic drugs include digoxin as first choice and sotalol, flecainide, amiodarone, verapamil, procainamide as second choice. ${ }^{2,11}$ Studies show that there is a significantly better response to sinus rhythm prenatally when digoxin is used in non-hydropic foetuses (80\%), compared with $43 \%$ in hydropic foetuses. ${ }^{8,12}$

It is important to remember that spontaneous conversion to sinus rhythm does happen in fetal AF postnatally, although this was not observed in our case. ${ }^{13}$ The ECG done postnatally confirmed the neonate was still in AF (Figure 1), hence the use of synchronised cardioversion which successfully resulted in sinus rhythm (Figure 2). Digoxin was continued as an antiarrhythmic prophylaxis. After sinus rhythm is achieved postnatally, one may monitor to see if there is recurrence with AF before instituting prophylaxis, or electively treat for 6 months to 1 year. ${ }^{10}$ However, the risk of AF recurrence is very rare beyond the neonatal period. In our case, digoxin was given for the first 28 days and to be continued for the first 6 months at least with follow up.

\section{Conclusion}

Fetal AF is a serious and threatening second commonest fetal tachyarrhythmia with an associated mortality rate of $10 \%$. Adequate diagnosis, awareness of association including fetal hydrops and cardiac anomaly, and multidisciplinary team involvement often ensure optimal outcome. Postnatal cardioversion a successful way of achieving sinus rhythm and antiarrhythmic prophylaxis is often necessary especially for the neonatal period. It is essential fetal AF is not managed as 'fetal distress' by general obstetricians and midwives. Call for help when in doubt.

\section{Author contribution}

Dr Nnadozie Igbokwe, the lead author wrote the manuscript, did literature review, got patient perspective and consent, and did the final editing.

Dr Aisha F Ibrahim summarised the clinical case notes and edited the final script.

Dr Samy Mutalab contributed to the discussion and key message.

Dr Oonagh Cleland suggested the case report and approved the final manuscript.

\section{Conflict of interest}

No conflict of interest declared.

\section{Acknowledgement}

Special acknowledgement to the patient for writing a very detailed patient perspective, and giving consent for the case publication.

\section{Ethical consideration}

A written informed consent was obtained from the patient.

\section{References}

1. Choi SH, Lee KH, Sohn CS, et al . A Case of Fetal Atrial Flutter with Hydrops Fetalis. J Korean Pediatr Soc. 1993 Aug 15;36(8):1165-70. 
2. Maeno Y, Hirose A, Kanbe T, et al . Fetal arrhythmia: prenatal diagnosis and perinatal management. $J$ Obstet Gynaecol Res . 2009 Aug;35(4):623-9.

3. Rauf M, Sevil E, Ayse B, et al. A case of fetal atrial flutter treated successfully by cardioversion in the postnatal period.Biomed Res 2017;28(7).

4. Wacker-Gussmann Annette, Strasburger Janette F., Srinivasan Sharda, et al . Fetal Atrial Flutter: Electrophysiology and Associations With Rhythms Involving an Accessory Pathway. J Am Heart Assoc . $5(6)$.

5. Sengheiser CJ, Channer KC. Recurrent atrial flutter and fibrillation in pregnancy. Case Rep . 2011 Jun 3;2011:bcr1220103589.

6. Wójtowicz-Marzec M, Wysokińska B, Respondek-Liberska M. Successful treatment of neonatal atrial flutter by synchronized cardioversion: case report and literature review. BMC Pediatr. 2020 Aug 5;20(1):370.

7. Miyoshi T, Maeno Y, Sago H, et al . Antenatal antiarrhythmic treatment for fetal tachyarrhythmias: a study protocol for a prospective multicentre trial. BMJ Open. 2017 Aug 1;7(8):e016597.

8. Krapp M, Kohl T, Simpson JM, et al . Review of diagnosis, treatment, and outcome of fetal atrial flutter compared with supraventricular tachycardia. Heart. 2003 Aug 1;89(8):913-7.

9. Wu T-H, Huang L-C, Ho M, et al . Fetal atrial flutter: a case report and experience of sotalol treatment. Taiwan J Obstet Gynecol. 2006 Mar;45(1):79-82.

10. Lisowski LA, Verheijen PM, Benatar AA, et al . Atrial flutter in the perinatal age group: diagnosis, management and outcome. J Am Coll Cardiol . 2000 Mar 1;35(3):771-7.

11. Takei K, Morikawa M, Cho K, et al . Resolution of tachyarrhythmia-related fetal hydrops after corticosteroid administration for fetal lung maturation. Case Rep. 2015 Nov 3;2015:bcr2015211948.

12. Api O, Carvalho JS. Fetal dysrhythmias. Best Pract Res Clin Obstet Gynaecol . 2008 Feb;22(1):31-48.

13. Sivakumar S. Atrial flutter in preterm babies. Arch Dis Child - Fetal Neonatal Ed . 2004 Nov 1;89(6):F564-F564.

\section{Hosted file}

Fetal AF images.pdf available at https://authorea.com/users/388714/articles/508478successful-management-of-fetal-atrial-flutter-at-term-pregnancy-with-postnatal-

electrocardioversion 